\title{
Validity of Dietary Diversity Score as an Indicator of Nutrient Adequacy among Older Adults in Pasay City, Philippines
}

\author{
Charlene Bridgitte C. Cokieng, ${ }^{1}$ Louie Amado R. Gutierrez, ${ }^{1}$ Angelica Noelle P. Manaloto, ${ }^{1}$ \\ Janel Priscilla C. See, ${ }^{1}$ Jolene Hannah C. Tan ${ }^{1}$ and Ernani R. Bullecer ${ }^{2}$ \\ ${ }^{1}$ College of Public Health, University of the Philippines Manila \\ ${ }^{2}$ Department of Nutrition, College of Public Health, University of the Philippines Manila
}

\begin{abstract}
Objective. The study was conducted to determine the validity of Dietary Diversity Score (DDS) as an alternative indicator for nutrient adequacy among older adults in Pasay City, Philippines.

Methods. An analytic cross sectional study design was used on a representative sample of older adults $(n=82)$ in all barangays under the Doña Marta Health Center in Pasay City. A 5-stage multiple pass single 24-hour food recall was performed to determine the dietary intake of each respondent. Subject's nutrient intake was obtained using the FCT+Menu Eval software. Nutrient Adequacy Ratio (NAR) was then computed using the ratio of subject's nutrient intake to the Recommended Energy and Nutrient Intakes (RENI) for Filipinos. The Mean Adequacy Ratio (MAR) was then obtained by computing for the average of the NARs, expressed as a ratio ranging from $0-1$. Dietary Diversity Score was calculated using the DDS Questionnaire recommended by the FAO Guideline. For the statistical analysis, Pearson's correlation was used to determine the relationship between MAR and DDS. ROC curve analysis was done to determine the most appropriate cut-off points for using DDS among the older adults.
\end{abstract}

Results. The selected older adults of Pasay City had a mean DDS of $4.15(0.14)$ and a mean MAR of $0.64(0.02)$. There was a significant and strong correlation between MAR and DDS $(r=$ $0.519 ; \mathrm{P}<0.0001)$. When maximizing sensitivity and specificity, the best cut-off points for achieving a MAR equivalent to both 0.5 and 0.7 were between DDS 4 and 5 .

Conclusion. DDS may be used as an indicator of nutrient adequacy among the randomly selected older adults.

Key Words: dietary diversity score, nutritional adequacy, older adults, mean adequacy ratio, nutrient adequacy ratio

\footnotetext{
Corresponding author: Ernani R. Bullecer, RND, MPH

Department of Nutrition

College of Public Health

University of the Philippines Manila

625 Pedro Gil Street, Ermita, Manila 1000 Philippines

Telephone: +6325255858

Fax no.: +632 5211394

Email: nans_bullecer@yahoo.com
}

\section{Introduction}

In latest years, there has been a strident rise in the number of older persons worldwide. ${ }^{1}$ The percentage of the population aged 60 and over, is also escalating each year. ${ }^{2}$ By the year 2025, the world will host 1.2 billion people aged 60 and over and rising to 1.9 billion in $2050 .^{3}$ In the Philippines, the proportion of the older adults is growing. According to the NSO census of 2010, there has been an increase of the elderly population living in the Philippines from $6 \%$ in the year 2000 to $6.8 \%$ in the year $2010 .^{4}$

The demographic transition with ageing of the population is a global phenomenon which demands international, national, regional and local action. 5,6 In recent years there has been an increasing international awareness of health issues relating to aging populations. ${ }^{7}$ Traditional perceptions of old age have been challenged during the past few years and it is important that older adults are not taken as a burden on society, but rather as an asset. ${ }^{6,7}$

The potential contribution to development efforts, the need for special care as well as the health and social services needed to care for the elderly make the growing older population a demographic concern, prompting a need to improve the quality of life of the said population. Thus, overall health is fundamental in the elderly and one of the means of achieving good health is through ensuring proper nutritional intake.

According to a study by Torheim et al. in 2004, dietary deficit is usually the concern in developing countries. Also, nutrient adequacy alone is commonly the means to which dietary quality is referred to. However, the frequently used methods for calculating the nutrient adequacy requires quantifying the intake of nutrients and this has been found to be expensive, time consuming, and associated with numerous methodological challenges in developing countries. $^{8}$

Nowadays, health workers need a more effective method for monitoring the nutrient quality of the people. They need indicators or methods that are rapid, efficient, inexpensive and simple enough for estimating nutrient adequacy suitable for large field assessments.

Since food is rarely eaten by itself as it is usually conjugated with other food items, trends in dietary 
assessment have moved from individual food items utilized by conventional methods such as the 24 -hour food recall and food weighing method to food groups put to use by other indicators such as dietary diversity score or DDS.

Across the years, a number of studies both foreign and local have been done stating that DDS may be used as an alternative indicator for nutrient adequacy among infants, children, adolescents and adults, but only a limited number of local ones delved into the older adults as the study group. Thus, this study aims to validate DDS as an alternative indicator for nutrient adequacy for selected older adults.

\section{Materials and Methods}

\section{Subjects}

The study participants included 82 residents aged 60 years and over from all eight barangays under the coverage of Doña Marta Health Center in Pasay City. A stratified random sampling design, which uses barangays as the stratification variable and the older adults as the elementary unit was employed in the study. An informed consent was obtained from the respondents who were randomly selected for the study.

\section{Dietary Intake}

Data was collected through the 5-stage multiple-pass 24hour food recall method. The 5-stage multiple-pass approach helped in the total recall of all foods consumed. At stage one, participants or the person caring for them, if accessible, was asked by an interviewer to enumerate the foods and beverages that the respondent had consumed in the past 24hours both at home and outside, including any vitamin and mineral supplements. The second stage commenced with a list of 9 food categories enumerated to facilitate recall of the person being interviewed for possible foods missed out during the first stage. The recorded foods and beverages from stages one and two were then recounted during the third stage and the interviewee was asked to recall the time they were consumed and also the eating occasion (e.g. breakfast, lunch, dinner, etc.). At the fourth recount, clarifications on listed foods were done when they may be eaten in combination (e.g. bread and spread for sandwiches) or if there were any leftovers or second helpings. Other descriptions, such as the brand name (where applicable) and the amounts of each food consumed in household measurements were also reported. To help in the recollection of portion sizes, food models were provided. During the fifth and final stage, a last review of the recorded foods and beverages was done. Probing was also done to ensure no other food or beverage consumed during the previous 24 hours was forgotten. ${ }^{9}$

\section{Nutrient Analysis}

Nutrient analysis was done to determine the nutrient intake of the respondents using the FCT + Menu Eval software developed by the Food and Nutrition Research Institute, Department of Science and Technology (FNRIDOST), Philippines, 2002. The analyses of the software were limited only to the 7 micronutrients which are Vitamins A, C, thiamin, riboflavin, niacin, calcium, and iron, as well as energy and protein.

\section{Nutrient Adequacy Ratio}

Nutrient adequacy of the diet was determined using nutrient adequacy ratio for each micronutrient, energy and protein. NAR was calculated by the following formula:

\section{$\mathrm{NAR}=\frac{\text { Actual nutrient intake of nutrient (per day) }}{\text { Recommended daily allowance of the nutrient }}$}

\section{Mean Adequacy Ratio}

The mean adequacy ratio (MAR) was calculated as a measure of the adequacy of the overall diet, where MAR is the sum of each NAR (truncated at 1) divided by the number of nutrients as shown below.

\section{MAR $=\frac{\sum \text { NAR }(\text { each truncated at } 1)}{\text { number of nutrients }}$}

\section{Dietary Diversity Score}

After the food recall was completed, the interviewer filled in the food groups in the Dietary Diversity Questionnaire based on the information gathered from the food recall. Probing of the respondent for any food group that was not mentioned was done to clarify whether or not any food item from the particular food group was consumed.

The proposed number of food groups to be included was based on FAO Guidelines for individual DDS score which reflects the nutritional quality of the diet and the probability of micronutrient inadequacy. To calculate DDS, certain food groups from the sixteen food groups (Table 1) were aggregated to form the nine food groups as suggested by the FAO Guidelines for the individual score as shown in Table 2.

\section{Statistical Analyses}

Encoded data from MS Excel was analyzed by STATA version 12.0. Data was reported as mean \pm SD. Mean MAR was also calculated. Pearson correlation coefficient was used to measure the strength of linear relationship between mean MAR (y-axis) and different values of DDS (x-axis). The Receiver Operating Characteristics (ROC) curve analysis was used to test for sensitivity and specificity. This was used to acquire the DDS cut off points with optimum sensitivity and specificity using MAR as the standard. 
Table 1. Sixteen Food Groups

\begin{tabular}{cl}
\hline Number & \multicolumn{1}{c}{ Food group } \\
\hline 1 & Cereals \\
2 & White roots and tubers \\
3 & Vitamin A rich vegetables and tubers \\
4 & Dark green leafy vegetables \\
5 & Other vegetables \\
6 & Vitamin A rich fruits \\
7 & Other fruits \\
8 & Organ meat \\
9 & Flesh meats \\
10 & Eggs \\
11 & Fish and seafood \\
12 & Legumes, nuts, and seeds \\
13 & Milk and milk products \\
14 & Oils and fats \\
15 & Sweets \\
16 & Spices, condiments, beverages \\
\hline
\end{tabular}

Table 2. Nine Food Groups

\begin{tabular}{ll}
\hline \multicolumn{1}{c}{ Number } & \multicolumn{1}{c}{ Food group } \\
\hline 1,2 & Starchy staples $^{1}$ \\
4 & Dark green leafy vegetables \\
3,6 and red palm oil if & Other vitamin A rich fruits and $_{\text {applicable }}$ \\
5,7 & vegetables $^{3}$ \\
8 & Other fruit and vegetables \\
9,11 & Organ meat \\
10 & Meat and fish \\
12 & Eggs \\
13 & Legumes, nuts and seeds \\
\hline
\end{tabular}

${ }^{1}$ The starchy staples food group is a combination of Cereals and White roots and tubers.

${ }^{2}$ The other vitamin A rich fruit and vegetable group is a combination of vitamin A rich vegetables and tubers and vitamin A rich fruit.

${ }^{3}$ The other fruit and vegetable group is a combination of other fruit and other vegetables.

${ }^{4}$ The meat group is a combination of meat and fish

\section{Results and Discussion}

\section{Results}

The age and sex characteristics of the respondents are summarized in Table 3, while a graphical representation of the distribution is depicted in Figure 1. A total of 82 respondents were included in the study. It was observed that there were more females $(n=44)$ than males $(n=38)$. Regardless of sex, majority of them belonged to the 65-69 year old age bracket, comprising $32.92 \%$ of the respondents.

The mean age of the selected residents was $69 \pm 7$ years and was found to be comparable across sexes, with that among males being $66.66 \pm 6$ years while that among females being $70.20 \pm 7$ years. Statistical data showed that among the respondents, females were significantly older than males $(\mathrm{P}=0.0179)$.

Table 4 shows the average MAR and the mean NARs of individual nutrients in the diet of the respondents. The average MAR of the respondents regardless of sex is 0.64 , wherein males contributed a mean MAR of 0.63 while females a mean of 0.65 . Although MAR, which measures overall diet quality is slightly higher in females than in males, the values are not significantly different $(\mathrm{P}=0.5966)$.

Table 3. Summary of age and sex characteristics of elderly

\begin{tabular}{cccc}
\hline Age (years) & Male $(\mathrm{n}=\mathbf{3 8})$ & Female $(\mathrm{n}=\mathbf{4 4})$ & Total $(\mathrm{n}=\mathbf{8 2})$ \\
\hline \multirow{2}{*}{$60-64$} & $62.13(0.38)$ & $62.1(0.48)$ & $62.12(0.29)$ \\
& $(\mathrm{n}=15)$ & $(\mathrm{n}=10)$ & $(\mathrm{n}=25)$ \\
$65-69$ & $65.77(0.26)$ & $66.5(0.29)$ & $66.15(0.20)$ \\
& $(\mathrm{n}=13)$ & $(\mathrm{n}=14)$ & $(\mathrm{n}=27)$ \\
$70-74$ & $71.33(0.56)$ & $72.33(0.34)$ & $71.93(0.34)$ \\
& $(\mathrm{n}=6)$ & $(\mathrm{n}=9)$ & $(\mathrm{n}=15)$ \\
$74-79$ & $77(1)$ & $76.83(0.54)$ & $76.88(0.44)$ \\
& $(\mathrm{n}=2)$ & $(\mathrm{n}=6)$ & $(\mathrm{n}=8)$ \\
$80+$ & $82(2)$ & $85(0.84)$ & $84.14(0.91)$ \\
& $(\mathrm{n}=2)$ & $(\mathrm{n}=5)$ & $(\mathrm{n}=7)$ \\
Total & $66.66(0.92)$ & $70.20(1.11)$ & $68.56(0.75)$ \\
& $(\mathrm{n}=38)$ & $(\mathrm{n}=44)$ & $(\mathrm{n}=82)$ \\
\hline
\end{tabular}

Values are expressed as mean (standard deviation)

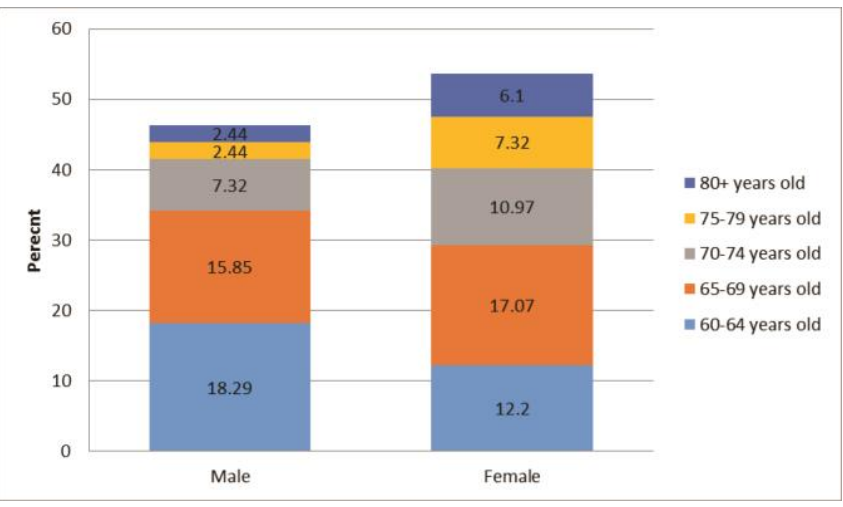

Figure 1. Percent distribution of respondents according to age and sex

Table 4. Mean MAR and mean NARs of the respondents

\begin{tabular}{cccc}
\hline Variable & Male $(\mathbf{n}=\mathbf{3 8})$ & Female $(\mathbf{n}=44)$ & Total $(\mathbf{n}=\mathbf{8 2})$ \\
\hline MAR & $0.63(0.03)$ & $0.65(0.03)$ & $0.64(0.02)$ \\
NAR Vitamin A & $0.51(0.05)$ & $0.62(.05)$ & $0.56(0.04)$ \\
NAR Vitamin C & $0.39(0.05)$ & $0.36(.05)$ & $0.38(0.04)$ \\
NAR thiamin & $0.50(0.04)$ & $0.48(.03)$ & $0.49(0.03)$ \\
NAR riboflavin & $0.52(0.04)$ & $0.65(0.04)$ & $0.59(0.03)$ \\
NAR niacin & $0.95(0.02)$ & $0.95(0.02)$ & $0.95(0.01)$ \\
NAR calcium & $0.48(0.04)$ & $0.50(0.04)$ & $0.49(0.03)$ \\
NAR iron & $0.72(0.04)$ & $0.71(0.05)$ & $0.71(0.03)$ \\
NAR energy & $0.77(0.04)$ & $0.80(0.03)$ & $0.79(0.02)$ \\
NAR protein & $0.88(0.08)$ & $0.75(0.03)$ & $0.75(0.02)$ \\
\hline
\end{tabular}

Values are expressed as mean (standard deviation)

NARs for most nutrients were found to be comparable between sexes. For the total study respondents, the nutrients with the top three highest NARs were: niacin (0.95), energy (0.79) and protein (0.75). All nutrients had an average of at least 0.5 except vitamin $C$, thiamin, and calcium. Vitamin C was found to have the lowest average NAR, being 0.39 in males and 0.36 in females, together with thiamin and calcium, whose average NARs were both found to be 0.49 .

Table 5 shows the mean DDS per food group and the total DDS in the diets of the elderly. Females (4.20) show a 
slightly higher mean DDS than males (4.05). However, the slight disparity was found to be not significant ( $\mathrm{P}=0.6647)$. The average DDS of all the respondents is 4.15. Regardless of sex, the food groups with the top three highest DDS are starchy staples, meat, fish and poultry and other fruits and vegetables while the food groups with the lowest DDS are organ meat and legumes, nuts and seeds. DDS of females for organ meat, legumes, nuts and seeds and milk and milk products are higher than those of males. On the other hand, males have higher DDS scores for dark green leafy vegetables and other Vitamin A rich vegetables, tubers, and fruits, meat, fish and poultry. Only results for milk and milk products are values statistically different $(\mathrm{P}=0.0363)$ between males and females.

Table 5. Mean and standard deviation of DDS per food group and total DDS in males and females

\begin{tabular}{lccc}
\hline \multirow{1}{*}{ Food groups } & \multicolumn{3}{c}{ Dietary Diversity Score } \\
\cline { 2 - 4 } & $\begin{array}{c}\text { Male } \\
(\mathbf{n}=\mathbf{3 8})\end{array}$ & $\begin{array}{c}\text { Female } \\
(\mathbf{n}=44)\end{array}$ & $\begin{array}{c}\text { Total } \\
\mathbf{( n = 8 2 )}\end{array}$ \\
\hline Starchy staple & $1(0)$ & $1(0)$ & $1(0)$ \\
Dark green and leafy & $0.38(0.08)$ & $0.30(0.07)$ & $0.33(0.05)$ \\
vegetables & & & \\
Other Vitamin A vegetables & $0.32(0.08)$ & $0.25(0.07)$ & $0.29(0.05)$ \\
and tubers & $0.78(0.69)$ & $0.68(0.07)$ & $0.73(0.05)$ \\
Other fruits and vegetables & $0.08(0.05)$ & $0.18(0.06)$ & $0.13(0.04)$ \\
Organ meat & $0.95(0.04)$ & $0.91(0.04)$ & $0.93(0.03)$ \\
Meat, fish and poultry & $0.27(0.07)$ & $0.30(0.07)$ & $0.29(0.05)$ \\
Eggs & $0.11(0.05)$ & $0.23(0.06)$ & $0.17(0.04)$ \\
Legumes, nuts and seeds & $0.16(0.06)$ & $0.36(0.07)$ & $0.27(0.05)$ \\
Milk and milk products & $4.05(0.22)$ & $4.20(0.19)$ & $4.15(0.14)$ \\
DDS & & & \\
\hline Values are expressed as mean (standard deviation) & & \\
&
\end{tabular}

Table 6 shows that males show a stronger correlation between NAR and DDS for calcium, protein, and all of the vitamins except niacin, while females show a stronger correlation for iron and energy. However, the only value which shows a significant correlation $(\mathrm{P}=0.0155)$ is that of riboflavin for both males and females.

Pearson correlation coefficients between NAR and DDS show that all correlations were significant with the exception of niacin, iron, energy and protein among males and calcium among females. MAR and DDS showed a significantly strong correlation $(\mathrm{r}=0.519, \mathrm{p}<0.0001)$ for the study population.

Figure 2 shows the relationship between DDS and NARs of energy, protein, calcium and iron. NAR reached 0.8 at a DDS of 3 in both energy and protein. For calcium, a NAR of 0.8 was achieved at a DDS of 8 , while a NAR of 0.8 was achieved at a DDS of 6 for iron. Figure 3 shows the relationship between DDS and NARs of vitamin A, vitamin $\mathrm{C}$, thiamin, riboflavin and niacin. NAR reached 0.8 at a DDS of 6 for vitamin A, at a DDS of 8 for vitamin C, at a DDS of 7 for thiamin, at a DDS of 6 for riboflavin and at a DDS of 2 for niacin. The following figures (Figure 4 and 5) indicate the sensitivity and specificity of the receiver-operator characteristic curves for MAR using DDS.

Table 6. Pearson correlation coefficients between nutrient adequacy ratio (NAR) of nutrients and dietary diversity score (DDS)

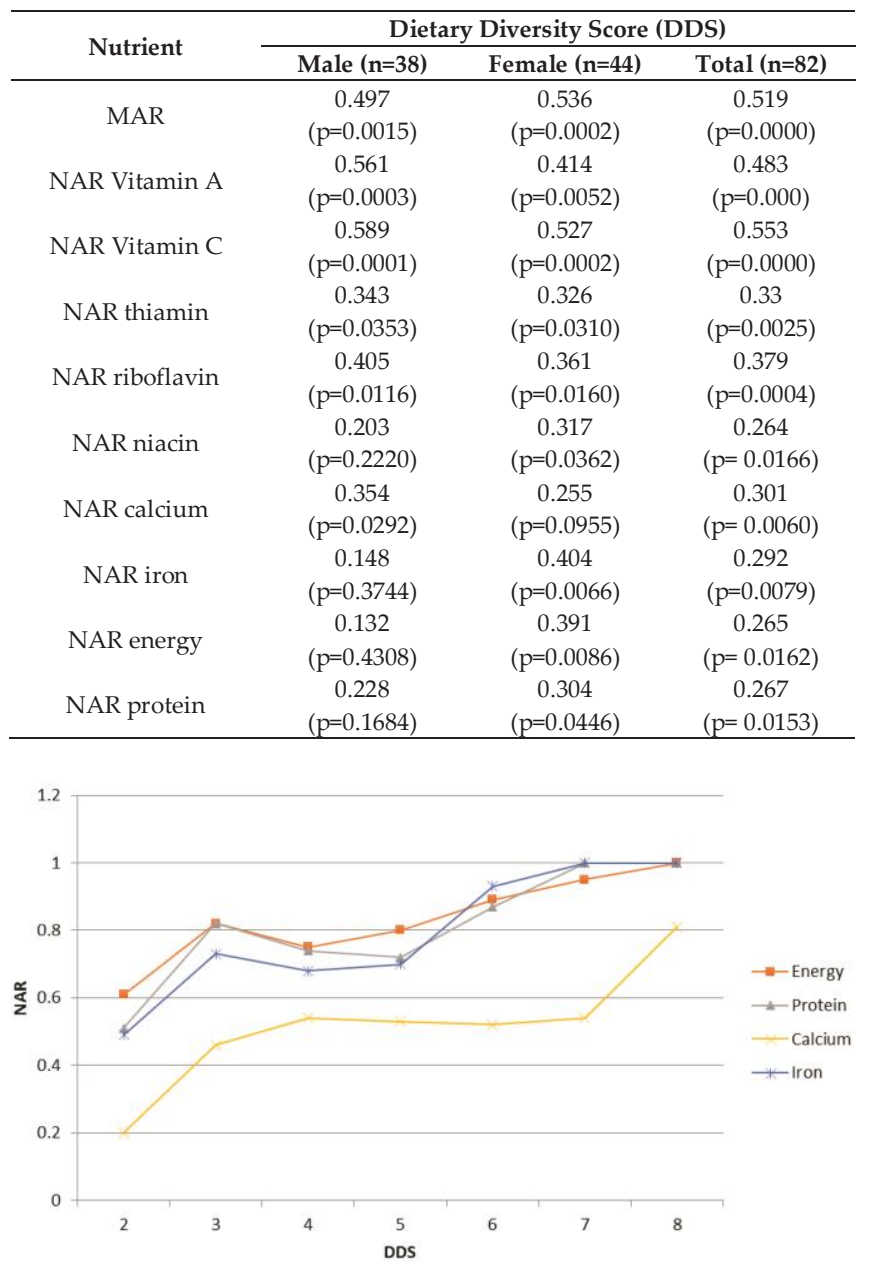

Figure 2. Mean nutrient adequacy ratio (NAR) of energy, protein and minerals at different levels of dietary diversity score (DDS)

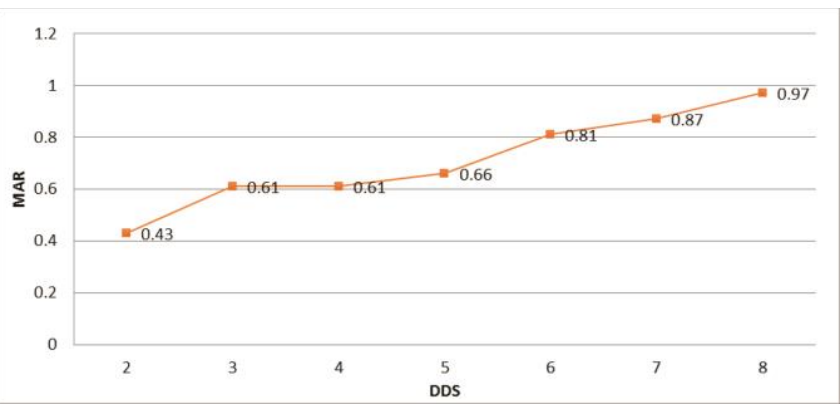

Figure 3. Mean nutrient adequacy ratio (NAR) of vitamins at different levels of dietary diversity score (DDS) 


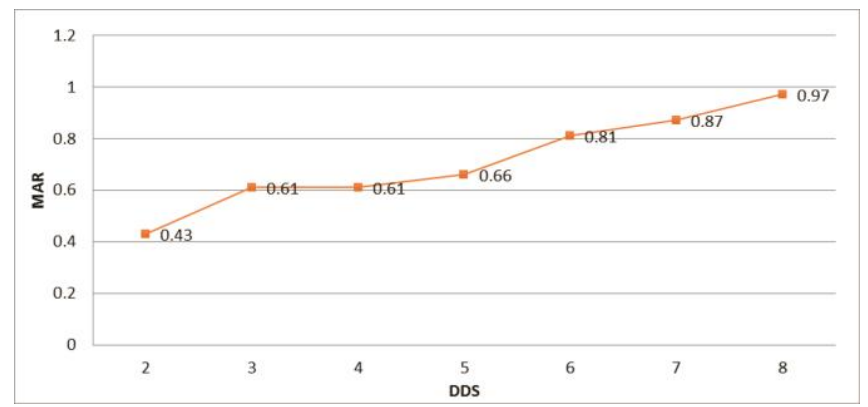

Figure 4. Mean adequacy ratio (MAR) for different levels of dietary diversity score (DDS)

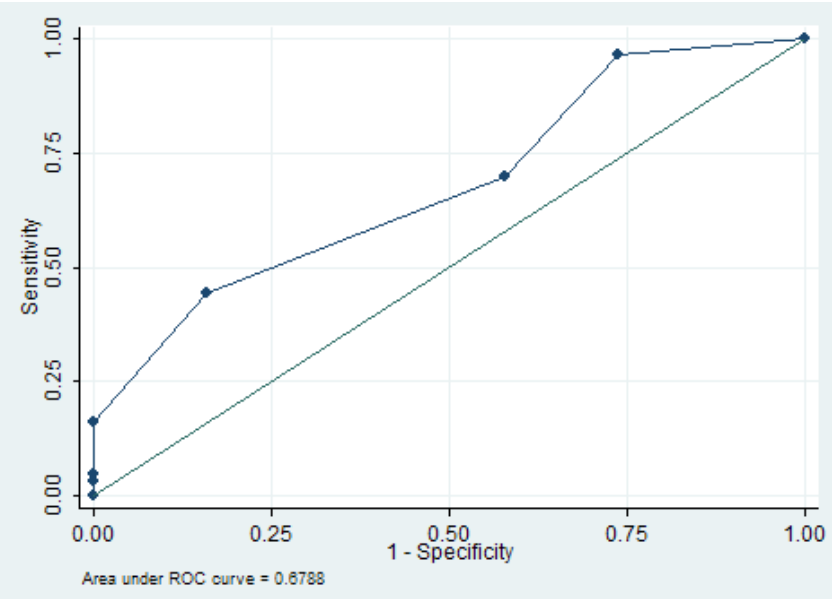

Figure 5. ROC curves showing sensitivity and specificity (\%) for $M A R \geq 0.5$

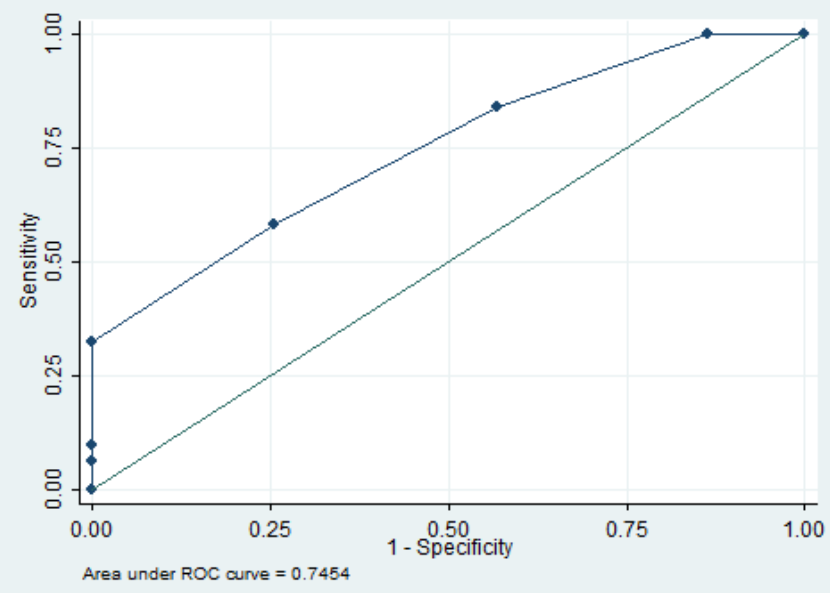

Figure 6. ROC curves showing sensitivity and specificity (\%) for $M A R \geq 0.7$

Mean MAR values for different values of DDS are presented in Figure 4. As MAR increases, DDS also increases. ROC curve analyses were used to determine the sensitivity and specificity of the different cut off points when
MAR $\geq 0.50$ and $M A R \geq 0.70$ as shown in Tables 7 and 8 , respectively. Tables 7 and 8 shows that DDS cut off points are directly proportional to specificity. On the other hand, low DDS cut off points show high sensitivity. If less than 0.5 MAR is selected as the cut off for sensitivity and greater than 0.5 as the cut off for specificity, then a DDS of 4 is most appropriate. It gives a sensitivity of $69.84 \%$ and a specificity of $42.11 \%$. If one selects less than 0.7 MAR as cut off for sensitivity, then a DDS of 5 is most appropriate. It gives a sensitivity of $58.06 \%$ and a specificity of $74.51 \%$.

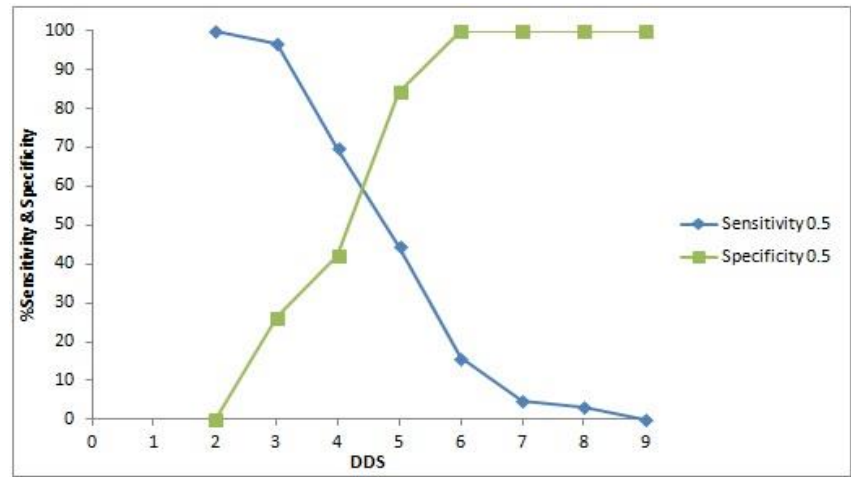

Figure 7. Sensitivity and specificity of DDS when MAR is 0.5 for elders

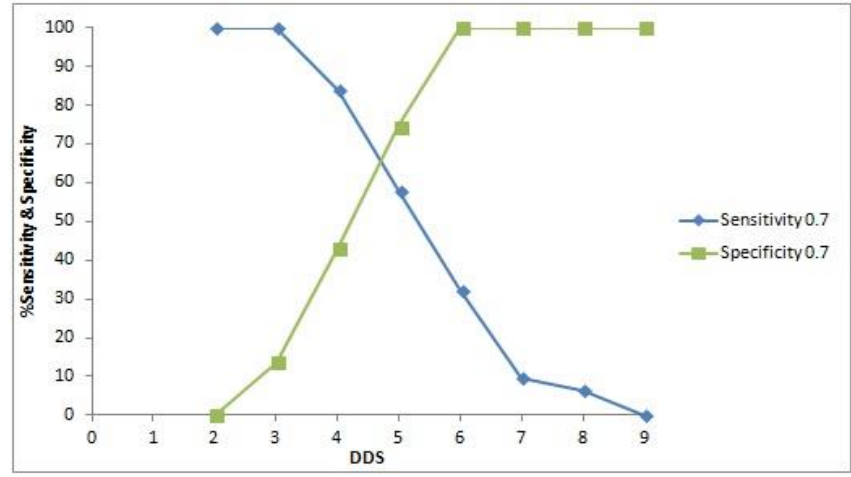

Figure 8. Sensitivity and specificity of DDS when MAR is 0.7 for elders

Table 7. DDS cut off points with corresponding sensitivity and specificity when MAR $\geq 0.50$

\begin{tabular}{ccc}
\hline DDS cut off points & Sensitivity & Specificity \\
\hline 1 & - & - \\
2 & $100 \%$ & $0 \%$ \\
3 & $96.83 \%$ & $26.32 \%$ \\
4 & $69.84 \%$ & $42.11 \%$ \\
5 & $44.44 \%$ & $84.21 \%$ \\
6 & $15.87 \%$ & $100 \%$ \\
7 & $4.76 \%$ & $100 \%$ \\
8 & $3.17 \%$ & $100 \%$ \\
9 & $0 \%$ & $100 \%$ \\
\hline
\end{tabular}


Table 8. DDS cut off points with corresponding sensitivity and specificity when $M A R \geq 0.70$

\begin{tabular}{ccc}
\hline DDS cut off points & Sensitivity & Specificity \\
\hline 1 & - & - \\
2 & $100 \%$ & $0 \%$ \\
3 & $100 \%$ & $13.73 \%$ \\
4 & $83.87 \%$ & $43.14 \%$ \\
5 & $58.06 \%$ & $74.51 \%$ \\
6 & $32.26 \%$ & $100 \%$ \\
7 & $9.68 \%$ & $100 \%$ \\
8 & $6.45 \%$ & $100 \%$ \\
9 & $0 \%$ & $100 \%$
\end{tabular}

\section{Discussion}

Numerous studies have shown that the intake of a variety of foods in the diet is necessary in acquiring essential nutrients as well as for the promotion of good health. This study demonstrates that DDS has a positive correlation with MAR for the randomly selected older adult population of Pasay City. Though this particular study population cannot be used to generalize all older adults in the country, much less the world, the results are parallel with those of foreign and local literature that were done on the different age groups such as infants, children, adolescents, and adults. This indicates that DDS may be used to predict the dietary quality of an individual in addition to estimating the nutrient adequacy of the said individual.

\section{Mean Nutrient Adequacy Ratio}

From the results, it can be seen that the average MAR of the study population is 0.64 , wherein MAR of male respondents is 0.63 and MAR of female respondents is 0.65 . Although MAR is slightly higher for females than in males, the values are not significantly different $(\mathrm{P}=0.5966)$.

\section{Dietary Diversity Score}

Female study participants show a slightly higher DDS (4.20) than males (4.05). However, there is no significant difference $(\mathrm{P}=0.6647)$. The average DDS of all the respondents is 4.15 and this is only slightly higher than the results obtained from previous studies done among Filipino adolescents and adults which observed a mean DDS of 3.94 and 3.63, respectively. In a study done on rural elderly in Sri Lanka, the mean DDS was found to be 4.4 using a 10 group DDS. ${ }^{10}$

DDS of female respondents for organ meat, legumes, nuts and seeds and milk and milk products are higher than those of males. On the other hand, male respondents have higher DDS scores for dark green leafy vegetables and other Vitamin A rich vegetables, tubers, and fruits, meat, fish and poultry. However, only the results for milk and milk products show a significant difference $(\mathrm{P}=0.0363)$ between males and females.

Findings also show that males have higher DDS for calcium, protein, and all of the vitamins except niacin.
Females have higher DDS for iron and energy. However, the only values which show a significant difference $(\mathrm{P}=0.0155)$ between males and females is that of riboflavin.

\section{Relationship between MAR and DDS}

MAR, which is the gold standard for the determination of nutrient adequacy, is positively correlated $(\mathrm{r}=0.519, \mathrm{p}<$ 0.0001 ) with DDS for a randomly selected older adults in Pasay City. Similar results were reported in other DDS studies among older adults, ${ }^{10}$ as well as the children and adults. ${ }^{11,15,16}$

There was a significant and positive correlation between DDS and most NARS for various nutrients as seen in Table 6 except for niacin, iron, energy and protein among males and calcium among females. The result of the study is consistent with other studies. ${ }^{16,18,19,20}$ The positive correlation of energy and protein intakes with DDS is contrary to the results obtained in other studies done among adults ${ }^{17}$ and older adults. ${ }^{18}$ The differences may be attributed to the number and dietary habits of the respondents and scoring methods.

Results revealed that dietary intake was highest with respect to niacin, energy, and protein and lowest with respect to vitamin $\mathrm{C}$, thiamin, and calcium. Moreover, it was observed that the study participants had the highest intakes of food under the groups starchy staples, meat, fish, and poultry, and other fruits and vegetables. Meanwhile, they had the poorest consumption of food under organ meat, legumes, nuts and seeds, and milk and milk products.

The low mean DDS (4.15) of the study participants may be attributed to the low consumption of organ meat (0.13), legumes, nuts and seeds (0.17), and milk and its products (0.27). This may be due to food availability, socioeconomic status, and personal preference. For instance, it has been a common belief that legumes increase susceptibility to arthritis, which is common myth among the older adults. Hence, they may have avoided legume consumption, which might have accounted for the low mean DDS for the food group. Such beliefs regarding legumes and arthritis have already been disproven in previous studies. ${ }^{21,22}$ The low consumption of milk and its products supported the low NAR for calcium (0.49). The fact that legumes, nuts, and seeds are known to be rich in thiamin has to be underscored to support the finding that thiamin placed as one of the lowest among the 9 nutrients in terms of average NAR. Thus, it can be claimed that the poor intake of food under that group is related to the observed low NAR for thiamin. It was recognized however that the consumption of starchy staples reached $100 \%$. All food recalls of the selected participants included white, long grain rice in their diets. According to the International Rice Research Institute (IRRI), white rice is rich in riboflavin, niacin, and energy, supporting the observed high NAR values for niacin and energy. This condition may be due to the dietary pattern of Filipinos, not only the older adults, of having a daily 
consumption of a rice based diet combined with other food commodities.

\section{Selection of DDS cut-off points}

The fourth objective of this study is to determine the sensitivity and specificity of DDS cut-off points using MAR as the standard for the selected older adult population.

The selection of DDS cut off point that most appropriately defines the MAR depends on the desired sensitivity and specificity. Sensitivity indicates the percentage of the respondents who are truly at risk of having inadequate diet and who are correctly classified as having low DDS, while specificity indicates the percentage of the respondents who are not at risk of having nutrient inadequacy and who are correctly classified as having high DDS. A more sensitive test will be less specific while a less sensitive test will be more specific. Thus, the selection of the cut off point will depend on the desired use of the DDS indicator.

For purposes of rapid screening for at risk individuals, it is preferred that a tool have higher sensitivity. With this, interventions will be addressed faster and financial considerations will be kept at minimum. Another rapid assessment tool with high specificity may be used consequently to minimize those who may have been falsely classified as having inadequate diet.

DDS cut off points in this study were tested for their sensitivity and specificity against definitions of a nutrient adequate diet. The nutrient adequate diet was defined to range between a MAR of 0.5 and 0.7 (50-70\% MAR) for nutrient intake.

The DDS with the best combination of sensitivity and specificity levels was selected to identify as many inadequate diets as really being inadequate (high sensitivity), but at the same time being able to identify respondents with a nutrient adequate diet (high specificity). From the results, the two selected DDS cutoff points which satisfy the above mentioned conditions for MAR 0.50 are DDS of 4 with $69.84 \%$ sensitivity and $42.11 \%$ specificity and DDS of 5 with $44.44 \%$ sensitivity and $84.21 \%$ specificity (Table 7).

Meanwhile, cutoff points which correspond to MAR 0.70 are DDS of 4 with sensitivity of $83.87 \%$ and specificity of $43.14 \%$ and a DDS of 5 with sensitivity of $58.06 \%$ and specificity of $74.51 \%$ (Table 7). Foreign studies conducted on older adults in Sri Lanka ${ }^{10}$ reported that a DDS of 4.5 corresponded to a MAR of 0.50 while another study done in South Africa reported that a DDS 6 of corresponded to a MAR of $0.70 .{ }^{14}$

Locally, a DDS of 4, regardless of whether it is a MAR of 0.5 or 0.7 , was consistent with the results of local studies done on adolescents and adults which also suggested that "DDS can be a good indicator of nutritional adequacy." 12,13

\section{Conclusion}

The results of the study revealed that the selected group of older adults have a relatively low MAR that indicates inadequate intake of essential nutrients. This was further confirmed by the low dietary diversity score (DDS) attained. The results acquired confirm that DDS may be used as an indicator of nutritional adequacy among selected older adults. Moreover, since DDS is easier and more rapid to use compared to the traditional methods available, it may be used as a substitute method especially during wide field assessments wherein field workers need a simple and effective method for assessing and monitoring the nutrient quality of the people. The use of DDS will allow swift identification of those older adults with inadequate dietary intakes which will both benefit the community as well as the health workers on field. It is recommended that further studies be done on other study populations such as institutionalized older adults, those living in rural settings or other urban sites using the same methodology which when compiled, may ultimately lead to the generalization of findings for the whole Filipino older adult population.

The use of DDS can indeed show diversity of diet components, however, in clinical settings and intervention programs the amount of food consumed must still be ascertained using nutrient adequacy and mean adequacy ratios.

\section{Acknowledgment and notes on Administrative Support}

The authors thank the following for their contribution in the study; Ms. Kim L. Cochon for the statistical analyses.

\section{References}

1. Hafez G, Bagchi K, Mahaini R. Caring for the elderly: a report on the status of care for the elderly in the Eastern Mediterranean Region. East Mediterr Health J. 2000; 6(4):636-43.

2. McMurdo ME. A healthy old age: realistic or futile goal? BMJ. 2000; 321(7269):1149-51.

3. World Population Prospects: The 2002 Revisions, Highlights. New York: United Nations Population Division. 2003.

4. National Statistics Office. The Age and Sex Structure of the Philippine Population: (Facts from the 2010 Census). NSO. [Online] August 30, 2012. Available from http://www.census.gov.ph/content/age-and-sexstructure-philippine-population-facts-2010-census.

5. World Health Organization (WHO)/ Eastern Mediterranean Region (EMR). Technical paper: Health care of the elderly in the Eastern Mediterranean Region: Challenges and Perspectives. 2003.

6. World Health Organization (WHO) Regional Office for Europe. Health and nutritional status of the elderly in the Former Yugoslav Republic of Macedonia: results of a national household survey. 2001.

7. Szucs TD. Future disease burden in the elderly: rationale for economic planning. Cardiovasc Drugs Ther. 2001; 15(4):359-61.

8. Torheim LE, Ouattara F, Diarra MM, et al. Nutrient adequacy and dietary diversity in rural Mali: association and determinants. Eur J Clin Nutr. 2004; 58(4):594-604.

9. Gibson R. Principles of nutritional assessment. New York: Oxford University Press. 1990. 
10. Rathnayake K, Madushani P, Silva K. Use of dietary diversity score as a proxy indicator of nutrient adequacy of rural elderly people in Sri Lanka. BMC Res Notes. 2012; 5:469.

11. Hatloy A, Torheim L, Oshaug A. Food variety- a good indicator of nutritional adequacy of the diet? A case study from an urban area in Mali, West Africa. Eur J Clin Nutr. 1998; 52(12):891-8.

12. Bullecer ER, Rabuco LB, Aninao DA, et al. Dietary Diversity Score as an indicator of nutritional adequacy of diets among 16-19 year-old adolescents. Acta Med Philipp. 2012; 46(1):27-33.

13. Bullecer ER, Pangan MRL, Arcegono MS, Agaton KSA. Validation of Dietary Diversity Score as an Indicator of Nutritional Adequacy of Diets among Selected Group of Adults in a University Campus in Manila, Philippines. JNDAP. 2012; 26(1\&2):21-31.

14. Oldewage Theron W, Kruger R. Impact of food aid on food variety and dietary diversity of an elderly community in Sharpeville, South Africa. J Nutr Health Aging. 2009; 13(4):300-8.

15. Arimond M, Wiesmann D, Becquey E, et al. Simple food group diversity indicators predict micronutrient adequacy of women's diets in 5 diverse, resource-poor settings. J Nutr. 2010; 140(11):2059S-69S.
16. Kant AK. Indexes of overall diet quality: a review. J Am Diet Assoc. 1996; 96(8):785-91.

17. Mirmiran P, Mohammadi F and Azizi F. Dietary diversity and its relation to diet quality in Tehran. Res Med. 2003; 27:33-41.

18. Marshall TA, Stumbo PJ, Warren JJ, et al. Inadequate nutrient intakes are common and are associated with low diet variety in rural, community-dwelling elderly. J Nutr. 2001; 131(8):2192-6.

19. Kant AK, Schatzkin A, Ziegler RG, et al. Dietary diversity in the US population, NHANES II, 1976-1980. J Am Diet Assoc. 1991; 91(12):152631.

20. Tucker KL. Eat a variety of healthful foods: old advice with new support. Nutr Rev. 2001; 59(5):156-8.

21. Saag KG, Choi H. Epidemiology, risk factors, and lifestyle modifications for gout. Arthritis Res Ther. 2006; 8(Suppl 1):1-7.

22. Alvarez-Lario B, Alonso-Valdivielso JL. Hyperuricemia and Gout: The role of diet. Nutr Hosp. 2014; 29(4):760-70.

\section{Acta Medica Philippina THE NATIONAL HEALTH SCIENCE JOURNAL

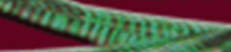

\section{Acta is now accepting membership}

Privileges of members:

- Allows you to submit articles for possible publication

- Have access to all the articles in the website (archives included) which can be downloaded and printed in pdf format

- Advertise your products/services in the available spaces of the website (for approval of the Editor-in-chief)

For details, please visit our website at www.actamedicaphilippina.com.ph or e-mail us at businessmanager@actamedicaphilippina.com.ph for any questions or queries. 